\title{
Application of Inverse Scattering Method to Problems of Differential Geometry
}

\author{
V. Zakharov ${ }^{1,2}$ \\ ${ }^{1}$ Landau Institute for Theoretical Physics, Moscow, 117334, Russia \\ 2 Department of Mathematics, University of Arizona, Tucson, AZ 85721, USA
}

\section{Introduction}

This article presents, in a brief form, the recent results on application of the Inverse scattering method to some problems of Differential geometry. A connection between the theory of Integrable systems and Differential geometry is not a new concept. The Sine-Gordon equation was introduced in the theory of surfaces of constant negative curvature around 1860. Actually, it should be called the "Bonnet equation". The Backlund transformations appeared in Differential geometry also in nineteenth century.

At present, it is established that some important integrable equations, found in the last three decades in the theory of solitons (Bullough-Dodd equation, DaweyStewartson stationary equation, etc.), have a geometrical interpretation. All of the equations mentioned above are in $1+1$ dimensions.

In this article we explore some geometrical applications of integrable systems in $2+1$ dimensions. The closest relative of the famous "three-wave equation",

$$
\begin{aligned}
& \frac{\partial \Psi_{1}}{\partial x_{1}}=i \Psi_{2} \Psi_{3}, \\
& \frac{\partial \Psi_{2}}{\partial x_{2}}=i \Psi_{1} \Psi_{3}^{*}, \\
& \frac{\partial \Psi_{3}}{\partial x_{3}}=i \Psi_{1} \Psi_{2}^{*},
\end{aligned}
$$

plays the central role in our study. Equation (1.1) describes a resonant interaction of three quasimonochromatic wave trains in a nonlinear media. This interaction 
is an induced Raman scattering of the wave 1 to waves 2 and 3 , and the inverse process. There is a special reason to present this article to this particular collection of papers. One of the pioneers in the study of system (1.1) was David Kaup [1]. (See also [2], [3].)

In this article we study the $n$-dimensional overdeterminated system,

$$
\begin{array}{cc}
\frac{\partial Q_{i j}}{\partial u^{j}}= & Q_{i j} Q_{j k}, \\
i, j, k=1, \ldots, n, & i \neq j \neq k .
\end{array}
$$

This system is nontrivial if $n \geq 3$. For $n=3$, equation (1.1) is a special case of (1.2), if $Q_{i j}$ is a complex-valued matrix. We will study mostly the case of real-valued $Q_{i j}$.

Equation (1.2) have appeared in Differential geometry in the middle of last century, long before the concept of Raman scattering was formulated. It's origin was connected with the problem of classification of $n$-orthogonal curvilinear coordinate systems in $R^{n}$. This problem, which was formulated almost two hundred years ago, was considered for a long time as one of the central in Differential geometry. In 1910, Gaston Darboux devoted to this problem (to the case $n=3$ only), the monogragh of 546 pages [4]. If an expert on the Inverse scattering technique looks into this book, he will be surprised: how many formulae are familiar to him!

After the First World War, the problem of $n$-orthogonal coordinate systems was almost forgotten. An interest to this problem was revived ten years ago, when Dubrovin and Novikov [5, 6], then Tzarev [7], have published their papers on integrable ststems of hydrodynamic type. The complete solution of this problem was found only in 1998 [8].

In the theory of $n$-orthogonal coordinate systems, equation (1.2) is considered together with the additional constrain:

$$
E_{i j}=\frac{\partial Q_{i j}}{\partial u^{j}}+\frac{\partial Q_{j i}}{\partial u^{i}}+\sum_{i \neq j \neq k} Q_{i k} Q_{j k}=0 .
$$

This constrain is a "reduction" imposed on (1.2). However, equation (1.2) itself has a nice geometrical interpretation. It describes $n$-dimensional Riemann spaces of diagonal curvature $[9,10]$.

The spaces of diagonal curvature include very interesting class of Riemann spaces: $2-D$ surfaces, spaces of constant curvature, and spaces of flat connection. They all are defined by the reduction more complicated than (1.3). In this article we will show how the Inverse scattering method could be implemented for description of the spaces of flat connection. We will show also that the closure of 
this class of spaces coincides with the whole class of the diagonal curvature. This question was formulated to the author by E. Ferapontov [11]. In this article we will use the Inverse scattering method in the form of "dressing method" $[2,9,12$, $13]$.

We will discuss also one important problem. Some classes of the Einstein spaces (solutions of Einstein equations of general relativity) belong to the class of spaces of diagonal curvature. The Schwartzshield's metric around a black hole is in this list. Can one find the class of reduction, which separates the Einstein spaces from the whole class of spaces of diagonal curvature? This extremely interesting question is still unanswered.

\section{$2 \quad N$-orthogonal coordinate systems}

The problem of classification of $n$-orthogonal curvilinear coordinate systems was formulated by Dupin and Binet in 1810. The problem is the following: Find in $R^{n}$ all coordinate systems,

$$
\begin{aligned}
& u^{i}=u^{i}\left(x^{1}, \ldots, x^{n}\right), \quad i=1, \ldots, n \\
& \operatorname{det}\left\|\frac{\partial u^{i}}{\partial x^{j}}\right\| \neq 0,
\end{aligned}
$$

satisfying the condition of orthogonality,

$$
\sum_{k=1}^{n} \frac{\partial u^{i}}{\partial x^{k}} \frac{\partial u^{j}}{\partial x^{k}}=0, \quad i \neq j .
$$

In virtue of (2.2) one can resolve equations (2.1),

$$
x^{i}=x^{i}\left(u^{1}, \ldots, u^{n}\right),
$$

and introduce Lamé coefficients,

$$
H_{i}^{2}=\sum_{k}\left(\frac{\partial x^{i}}{\partial u^{k}}\right)^{2}
$$

In the coordinate system $u^{i}$ the metric tensor in $R^{n}$ is diagonal,

$$
d s^{2}=\sum_{i=1}^{n} H_{i}^{2}\left(d u^{i}\right)^{2},
$$


and Christoffel's coefficients for the Levi-Civita connection are the following:

$$
\begin{aligned}
\Gamma_{l m}^{i} & =0(i \neq l \neq m) \\
\Gamma_{i l}^{i} & =\frac{1}{H_{i}} \frac{\partial H_{i}}{\partial u^{l}} \\
\Gamma_{l l}^{i} & =-\frac{H_{l}}{H_{i}^{2}} \frac{\partial H_{l}}{\partial u^{i}} .
\end{aligned}
$$

One can calculate the elements of Riemann curvature tensor $R_{i j k l}$. They are:

$$
\begin{aligned}
R_{i j, k l} & =0, \quad i \neq j \neq k \neq l \\
R_{i k, j k} & =-H_{i} H_{j}\left(\frac{\partial Q_{i j}}{\partial u^{k}}-Q_{i k} Q_{k j}\right), \quad i \neq j \neq k, \\
R_{i j, i j} & =-H_{i} H_{j} E_{i j}, \quad i \neq j,
\end{aligned}
$$

where rotation coefficients $Q_{i j}$ are

$$
Q_{i j}=\frac{1}{H_{j}} \frac{\partial H_{i}}{\partial u^{j}}, \quad i \neq j
$$

and

$$
E_{i j}=\frac{\partial Q_{i j}}{\partial u^{j}}+\frac{\partial Q_{j i}}{\partial u^{i}}+\sum_{k \neq i, j} Q_{i k} Q_{j k}, \quad i \neq j .
$$

As far as $R^{n}$ is flat, the Riemann curvature tensor is identically equal zero. Hence $Q_{i j}$ satisfy the following two systems of equations:

$$
\begin{aligned}
\frac{\partial Q_{i j}}{\partial u^{k}} & =Q_{i k} Q_{k j}, \\
E_{i j} & =\frac{\partial Q_{i j}}{\partial u^{j}}+\frac{\partial Q_{j i}}{\partial u^{i}}+\sum_{k \neq i, j} Q_{i k} Q_{j k}=0 .
\end{aligned}
$$

If $Q_{i j}$ are known, the Lamé coefficients $H_{i}$ can be found as solutions of the linear system

$$
\frac{\partial H_{i}}{\partial u^{j}}=Q_{i j} H_{j}, \quad i \neq j
$$

Two different solutions of system (2.15), $H_{i}$ and $\tilde{H}_{i}$, are Combescure equivalent, $H_{i} \leftrightarrow \tilde{H}_{i}$, at the same $Q_{i j}$. The Lamé coefficients satisfy to Gauss-Lamé equations,

$$
\frac{\partial^{2} H_{i}}{\partial u^{l} \partial u^{m}}=\frac{1}{H_{l}} \frac{\partial H_{l}}{\partial u^{m}} \frac{\partial H_{i}}{\partial u^{l}}+\frac{1}{H_{m}} \frac{\partial H_{m}}{\partial u^{l}} \frac{\partial H_{i}}{\partial u^{m}},
$$


and to the additional system,

$$
\frac{\partial}{\partial u^{l}} \frac{\partial H_{i}}{H_{l} \partial h^{l}}+\frac{\partial}{\partial u^{i}} \frac{1}{H_{i}} \frac{\partial H_{l}}{\partial u^{l}}+\sum \frac{1}{\left(H_{m}\right)^{2}} \frac{\partial H_{i}}{\partial u^{m}} \frac{\partial H_{l}}{\partial u^{m}}=0,(i \neq l) .
$$

One can construct the adjoint linear system,

$$
\frac{\partial \Psi_{i}}{\partial u^{k}}=Q_{i k} \Psi_{k}, \quad i \neq k,
$$

where $\Psi_{i}$ are adjoint Lamé coefficients. They satisfy to equation (2.16) and to the additional system,

$$
\frac{\partial}{\partial u^{l}} \frac{\partial \Psi_{i}}{\Psi_{l} \partial u^{l}}+\frac{\partial}{\partial u^{i}} \frac{1}{\Psi_{i}} \frac{\partial \Psi_{l}}{\partial u^{i}}+\frac{1}{\Psi_{i} \Psi_{l}} \sum_{n \neq i, j} \frac{\partial \Psi_{m}}{\partial u^{i}} \frac{\partial \Psi_{m}}{\partial u^{j}}=0
$$

Both systems (2.15), (2.18) are overdeterminated and are compatible in virtue of (2.13).

Note that

$$
\frac{\partial}{\partial u^{i}} H_{k} \Psi_{k}=\frac{\partial}{\partial u^{k}} H_{i} \Psi_{i}
$$

hence

$$
H_{k} \Psi_{k}=\frac{\partial h}{\partial u^{k}} .
$$

One can check that the potential $h$ satisfies the system of Laplace equations,

$$
\begin{aligned}
\frac{\partial^{2} h}{\partial u^{k} \partial u^{l}} & =\frac{1}{H_{k}} \frac{\partial H_{k}}{\partial u^{l}} \frac{\partial h}{\partial u_{k}}+\frac{1}{H_{l}} \frac{\partial H_{l}}{\partial u^{k}} \frac{\partial h}{\partial u^{l}}, \\
\frac{\partial^{2} h}{\partial u^{k} \partial u^{l}} & =\frac{1}{\Psi_{k}} \frac{\partial \Psi_{k}}{\partial u^{l}} \frac{\partial h}{\partial u_{k}}+\frac{1}{\Psi_{l}} \frac{\partial \Psi_{l}}{\partial u^{k}} \frac{\partial h}{\partial u^{l}} .
\end{aligned}
$$

Systems (2.21), (2.22) are overdeterminated linear equations imposed on the potential $h$. They can be treated as an analog of the "Lax pair" for Gauss-Lamé equations (2.16).

Equation (2.21) can be rewritten as follow:

$$
\frac{\partial^{2} h}{\partial u^{k} \partial u^{l}}=\Gamma_{k l}^{k} \frac{\partial h}{\partial u^{k}}+\Gamma_{l k}^{l} \frac{\partial h}{\partial u^{l}},
$$

and one can prove that functions $x^{i}=x^{i}\left(u^{1}, \ldots, u^{n}\right)$ are solutions of (2.23):

$$
\frac{\partial^{2} x^{i}}{\partial u^{k} \partial u^{l}}=\Gamma_{k l}^{k} \frac{\partial x^{i}}{\partial u^{k}}+\Gamma_{l k}^{l} \frac{\partial x^{i}}{\partial u^{l}} .
$$


Moreover, they satisfy the equations

$$
\frac{\partial^{2} x^{i}}{\left(\partial u^{l}\right)^{2}}=\sum_{k} \Gamma_{l l}^{k} \frac{\partial x^{l}}{\partial u^{k}} .
$$

Equations (2.24), (2.25) stem from the fact that a straight line defined by conditions $x^{i}=c^{i}=$ const, $i \neq l$, is the geodesic line in $R^{n}$.

We see now that the problem of description of $n$-orthogonal coordinate systems can be solved in the following several steps.

1. Solve system (2.13).

2. Find the solutions for linear system (2.15); on this stage one finds all Combescure equivalent metrics connected with the given rotation coefficients $Q_{i j}$ satisfying equation (2.14).

3. Solve the adjoint system (2.18).

4. Integrate the relation (2.20); on this stage one finds the general solution of Laplace equations (2.21), (2.22).

5. Find the solutions of (2.13), satisfying additional conditions (2.11).

6. Find $x^{i}\left(u^{1}, \ldots, u^{n}\right)$, the array of solutions of (2.21) and (2.24) satisfying additional conditions (2.25). In virtue of the Bonnet theorem, $x^{i}$ are defined uniquely up to motions in $R^{n}$. Note, that systems (2.24), (2.25) are compatible only if equation (2.11) is satisfied.

The first five points of this program pertain to the intrinstic geometry of $R^{n}$ in a new curvilinear coordinate system. On this stage $R^{n}$ appears as a flat Riemann space with diagonal metric (2.6). The last point realizes embedding of this space to Cartesian coordinate system $x^{i}\left(u^{1}, \ldots, u^{n}\right)$.

\section{Spaces of diagonal curvature}

In this chapter we introduce a new geometrical object - the space of diagonal curvature. By definition, it is a Riemann space of $n$ dimensions, $G^{n}$, satisfying the following two conditions:

Condition 1. One can introduce in $G^{n}$ (in some simple-connected domain) a diagonal coordinate system, such that the metric tensor $g_{i k}$ is diagonal,

$$
g_{i k}=H_{i}^{2} \delta_{i k}
$$


Condition 2. In this coordinate system the non-diagonal elements of Riemann's tensor are zero,

$$
R_{i k, j k}=0, \quad i \neq j \neq k .
$$

Note, that the diagonal coordinate system (3.1) could be introduced by many different ways. $G^{n}$ is the space of diagonal curvature if at least in one diagonal coordinate system condition (3.2) is satisfied.

The most trivial example of the space of diagonal curvature is a flat space $R^{n}$ or torus $R^{n} / Z^{n}$. In this case, description of diagonal metric tensors is exactly the problem of classification of $n$-orthogonal systems in $R^{n}$. One can display other examples of the spaces of diagonal curvature.

\section{Adjoint Lamé metrics.}

Let $H_{i}$ be Lamé coefficients for an $n$-orthogonal curvilinear in $R^{n}$ and $\Psi_{i}$ adjoint Lamé coefficients. Let us consider a Riemann space with the following metric:

$$
d s^{2}=\sum \Psi_{i}^{2}\left(d u^{i}\right)^{2}
$$

It is a space of diagonal curvature. However, it is not flat in a general case, because the additional constrain (2.29) imposed on $\Psi_{i}$ is different from condition (2.17) imposed on $H_{i}$. Only in a very special case of "Egorov's metric", when $Q_{i j}$ is a symmetric matrix, $Q_{i k}=Q_{k i}$, we will have

$$
H_{i}=\Psi_{i}, \quad H_{i}^{2}=\frac{\partial h}{\partial u^{i}},
$$

and conditions (2.16), (2.178) will coincide.

\section{Spaces of constant curvature.}

In the spaces of constant curvature, the Riemann's tensor is:

$$
R_{i j, k l}=\epsilon\left(g_{i k} g_{j l}-g_{i l} g_{j k}\right)
$$

Here $\epsilon$ is the curvature of space.

All such spaces admit diagonal metrics, $g_{i k}=H_{i}^{2} \delta_{i k}$. In this case,

$$
E_{i j}=\epsilon H_{i} H_{j}
$$

By a trivial rescaling one can get $\epsilon= \pm 1$.

\section{Spaces of flat connection.}

Spaces of flat connection, $G^{n, N}$, are generalizations of spaces of constant curvature. Let $H_{i}^{(k)}, k+1, \ldots, N$ be a set of Combescure equivalent metrics, corresponding to the given rotation coefficients $Q_{i j}$. In the space of flat connection 
$R_{i j, k j}=0$, and

$$
E_{i j}=\sum_{k=1}^{N} \epsilon_{k} H_{i}^{(k)} H_{j}^{(k)}, \quad \epsilon_{k}= \pm 1 .
$$

Spaces of flat connection appear in the following geometrical problem. Let us consider a special class of $n$-orthogonal curvilinear coordinates in $n+N$ dimensional Euclidean space $R^{n+N}$. Suppose, that coordinates in this space can be separated in two classes: $u^{1}, \ldots, u^{n}$ and $y^{1}, \ldots, y^{N}$; and the metric is

$$
d s^{2}=\sum_{i=1}^{n} H_{i}^{2}\left(d u^{i}\right)^{2}+\sum_{i=1}^{N}\left(d y^{i}\right)^{2} .
$$

In this case $H_{i}$ are linear functions on $y$,

$$
H_{i}=P_{i}+\sum_{a=1}^{N} H_{i}^{a} y^{a}
$$

where $P_{i}, H_{i}^{a}$ are functions on coordinates $u^{i}$ only.

One can introduce rotation coefficients,

$$
Q_{i j}\left(u^{1}, \ldots, u^{n}\right)=\frac{1}{P_{j}} \frac{\partial P_{i}}{\partial u^{j}},
$$

which satisfy the system of equations

$$
\frac{\partial Q_{i j}}{\partial u^{k}}=Q_{i k} Q_{k j}
$$

Hence the $n$-dimensional Riemann space $G^{n, N}$ with the metric

$$
d s^{2}=\sum_{i=1}^{n} H_{i}^{2}\left(d u^{i}\right)^{2}
$$

is the space of diagonal curvature. Moreover, $H_{i}^{a}$ satisfies the equations

$$
\frac{\partial H_{i}^{a}}{\partial u^{k}}=Q_{i k} H_{k}^{a}
$$

Thus all arrays $H_{i}^{a}, H_{i}^{b}$ are Combescure equivalent to each other and to $P_{i}$. One can easily check that

$$
E_{i j}=\frac{\partial Q_{i j}}{\partial u^{j}}+\frac{\partial Q_{j i}}{\partial u^{i}}+\sum_{k \neq i, j} Q_{i k} Q_{j k}=-\sum_{a} H_{i}^{a} H_{j}^{a} .
$$


It means that $G^{n, N}$ is a space of flat connection.

All two-dimensional Riemannian spaces (surfaces) are spaces of flat connection. Let $\Gamma$ be a surface in $R^{3}$. One can introduce coordinates $x_{1}, x_{2}$ on $\Gamma$ such that both the first and the second quadratic forms of the surface are diagonal,

$$
\begin{aligned}
& \omega_{1}=p^{2} d x_{1}^{2}+q^{2} d x_{2}^{2}, \\
& \omega_{2}=p A d x_{1}^{2}+q B d x_{2}^{2} .
\end{aligned}
$$

Coordinates $x_{1}, x_{2}$ are defined up to the trivial transformation $x_{1}=x_{1}\left(u_{1}\right), x_{2}=$ $x_{2}\left(u_{2}\right)$. The coefficients of these two quadratic forms $\omega_{1}, \omega_{2}$ cannot be chosen independently. They are connected by three nonlinear PDE's known as GaussCodazzi equations (GCE). These equations can be written in a nice and compact form after introducing new functions $\alpha, \beta$ :

$$
\alpha=\frac{1}{q} \frac{\partial p}{\partial x_{2}}, \quad \beta=\frac{1}{p} \frac{\partial q}{\partial x_{1}} .
$$

Thus

$$
\frac{\partial p}{\partial x_{2}}=\alpha q, \quad \frac{\partial q}{\partial x_{1}}=\beta p
$$

then

$$
\frac{\partial A}{\partial x_{2}}=\alpha B, \quad \frac{\partial B}{\partial x_{1}}=\beta A,
$$

and

$$
\frac{\partial \alpha}{\partial x_{2}}+\frac{\partial \beta}{\partial x_{1}}+A B=0
$$

Let us embed the surface $\Gamma$ in $R^{3}$. One can do this by constructing in vicinity of $\Gamma$ a special three-orthogonal coordinate system, such that

$$
d s^{2}=H_{1}^{2} d x_{1}^{2}+H_{2}^{2} d x_{2}^{2}+H_{3}^{2} d x_{3}^{2},
$$

where

$$
H_{1}=p+A x_{3}, \quad H_{2}=q+B x_{3}, \quad H_{3}=1 .
$$

Apparently $x_{3}$ is directed along the normal vectors to $\Gamma$.

One can check that rotation coefficients $Q_{i j}$ are:

$$
\begin{aligned}
& Q_{31}=Q_{32}=0 \\
& Q_{13}=A, \quad Q_{23}=B, \quad Q_{12}=a, Q_{21}=b
\end{aligned}
$$

The rotation coefficients $Q_{i j}$ satisfy the system (3.10), which is reduced now to system (3.17). Thus, a two-dimensional surface is the space of diagonal curvature. 
Moreover, comparing (3.16) and (3.17) one can see that $A^{2}, B^{2}$ can be treated as elements of an orthogonal metric that is Combescure equivalent to the elements of first quadratic form $p^{2}, q^{2}$. Hence equation (3.18) can be interpreted as a special case of equation (3.13), and $2-D$ surface is the space of flat connection. In a general case, equations (3.10), (3.12) and (3.13) can be called Gauss-Codazzi equations as well.

\section{Einstein spaces of diagonal curvature}

So far we discussed Riemann spaces of signature $(n, 0)$ only, but the whole theory can be extended to pseudo-Riemann spaces of arbitrary signature $(p, q)$. One has just to assume that some Lamé coefficients $H_{i}$ are pure imaginary. In this chapter we will discuss spaces of diagonal curvature $G^{(4)}$ satisfying Einstein equations of general relativity.

These equations read:

$$
\Lambda_{k}^{i}=\frac{8 \pi k}{c^{4}} T_{k}^{i},
$$

where $k$ is the gravity constant, $c$ is the light velocity, and

$$
\Lambda_{k}^{i}=R_{k}^{i}-\frac{1}{2} R \delta_{k}^{i}
$$

Here $R_{k}^{i}$ is the Ricci tensor, $R$ is a scalar curvature, and $T_{k}^{i}$ is an energy-momentum tensor. Thereafter we will use a system of units such that $k / c^{4}=1$. Thus,

$$
\Lambda_{k}^{i}=8 \pi T_{k}^{i}
$$

We will also use the covariant version of Einstein equations,

$$
R_{i k}-\frac{1}{2} R g_{i k}=8 \pi T_{i k}
$$

Due to the Bianchi identity, the Einstein's tensor $\Lambda_{k}^{i}$ satisfies the condition

$$
\Lambda_{k, i}^{i}=0 .
$$

In virtue of (4.1), the same condition is imposed on the energy-momentum tensor,

$$
T_{k, i}^{i}=0 .
$$

In general, (4.5) is the condition imposed on the energy-momentum spectrum. If one interprets Einstein equations in that broad sense, any Riemann space of 
signature $(1,3)$ can be treated as a solution of Einstein equations (Einstein space) for a proper energy-momentum tensor. Condition (4.4) is satisfied automatically.

Suppose that $g_{i k}$ is diagonal. In this case $g^{i k}=1 / H_{i}^{2} \delta_{i k}$, and

$$
R_{k l}=\frac{1}{H_{i}^{2}} R_{k i, l i} .
$$

In the space of diagonal curvature the Ricci tensor is diagonal too:

$$
\begin{aligned}
R_{k l} & =\tilde{R}_{k} \delta_{k l}, \\
\tilde{R}_{k} & =-H_{k} \sum_{i \neq k} \frac{E_{i k}}{H_{i}} .
\end{aligned}
$$

In the same way:

$$
\begin{aligned}
R_{l}^{k} & =R_{k} \delta_{l}^{k} \\
R_{k} & =-\sum_{i \neq k} \alpha_{i k}, \quad \alpha_{i k}=\frac{E_{i k}}{H_{i} H_{k}}
\end{aligned}
$$

and

$$
\Lambda_{k}^{i}=\Lambda_{k} \delta_{k}^{i}, \quad \Lambda=\sum \alpha_{l m}, \quad(l \neq i, m \neq i, l<m) .
$$

The energy-momentum tensor $T_{k}^{i}$ is diagonal as well:

$$
T_{k}^{i}=T_{k} \delta_{k}^{i}
$$

and Einstein equations can be written in the form:

$$
\Lambda_{k}=8 \pi T_{k}
$$

Here $T_{k}$ are arbitrary solutions of the following equation:

$$
\frac{\partial T_{k}}{\partial u^{k}}+\sum_{i \neq k} T_{i} \frac{\partial \ln H_{k}}{\partial u^{i}}=0 .
$$

If $\Lambda_{k}$ are components of the Einstein tensor for some space of diagonal curvature, equations (4.14) are satisfied automatically.

Suppose that $T_{k} \equiv 0$, and Einstein equations describe metrics in vacuum. In this case:

$$
\alpha_{12}=\alpha_{34}=\alpha, \quad \alpha_{13}=\alpha_{24}=\beta, \quad \alpha_{14}=\alpha_{23}=\gamma,
$$

and $\alpha+\beta+\gamma=0$. 
At the moment, one can enlist more than a dozen exact solutions of Einstein equations with a diagonal metric tensor. It is interesting that most of them describe spaces with a diagonal curvature. We present here a list of such spaces, that is far from being complete.

\section{1. "One-dimensional" metrics}

Let

$$
d s^{2}=-H_{0}^{2} d x_{0}^{2}+H_{1}^{2} d x_{1}^{2}+H_{2}^{2} d x_{2}^{2}+H_{3}^{2} d x_{3}^{2},
$$

where $H_{i}$ are functions on one variable only, suppose on $x_{0}$. In this case the only nontrivial rotational coefficients are

$$
Q_{i 0}=\frac{1}{H_{0}} \frac{\partial H_{i}}{\partial x_{0}}=Q_{i 0}\left(x_{0}\right)
$$

and apparently, all equations (3.10) are satisfied. In a general case, metric (4.16) presumes existence of matter. In vacuum, it turns to the Kasner metric:

$$
\begin{aligned}
& H_{1}=x_{0}^{2 p_{1}}, x_{2}=x_{0}^{2 p_{2}}, x_{3}=x_{0}^{2 p_{3}}, \\
& p_{1}+p_{2}+p_{3}=1, \quad p_{1}^{2}+p_{2}^{2}+p_{3}^{2}=1 .
\end{aligned}
$$

\section{Spherically-symmetric Einstein spaces}

In the theory of general relativity, this is a very important class of spaces, which includes the Schwartzshield's space outside of a black hole, the basic cosmological models, and Tolman's space describing the collapse of dust matter. In all these cases the metric is

$$
d s^{2}=-H_{0}^{2} d x_{0}^{2}+H_{1}^{2} d x_{1}^{2}+x_{1}^{2}\left(d x_{2}^{2}+\sin ^{2} x_{2} d x_{3}^{2}\right),
$$

where $H_{0}, H_{1}$ are functions on $x_{0}, x_{1}$ only.

In this case, the nonzero rotation coefficients are:

$$
\begin{aligned}
& Q_{01}=\frac{1}{H_{1}} \frac{\partial H_{0}}{\partial x_{1}}, Q_{10}=\frac{1}{H_{0}} \frac{\partial H_{1}}{\partial x_{0}}, Q_{32}=\cos x_{2}, \\
& Q_{21}=\frac{1}{x_{1}}, \quad Q_{31}=\frac{1}{x_{3}} \sin x_{2} .
\end{aligned}
$$

Among equations (3.10), the only one is non-trivial,

$$
\frac{\partial Q_{31}}{\partial x_{2}}=Q_{32} Q_{21}
$$

and is satisfied in virtue (4.20). 
Hence all the spherically symmetric Einstein spaces, both in vacuum and in the presence of matter, are spaces of diagonal curvature.

\section{Bianchi III model}

In this case,

$$
-d s^{2}=-d x_{0}^{2}+a^{2} e^{-2 m x_{3}} d x_{e}^{2}+b^{2} d x_{2}^{2}+c^{2} d x_{3}^{2},
$$

where $a, b, c$ are functions on $x_{0}$ only.

One can check that metric (4.22) describes the space of diagonal curvature if $c\left(x_{0}\right)=\lambda a\left(x_{0}\right),(\lambda$ is constant $)$.

\section{Bianchi V model}

In this model the metric has the form

$$
-d s^{2}=-d x_{0}^{2}+a^{2} e^{2 x_{3}} d x_{1}^{2}+b^{2} e^{2 x_{3}} d x_{2}^{2}+c^{2} d z^{2},
$$

where $a, b, c$ are functions on $x_{0}$ only.

This model describes the space of diagonal curvature if $a=\lambda_{1} c, b=\lambda_{2} c\left(\lambda_{1}, \lambda_{2}\right.$ are constants).

\section{Bianchi VI model}

Now

$$
-d s^{2}=d x_{0}^{2}+a^{2} e^{-2 m x_{3}} d x_{1}^{2}+b^{2} e^{2 x_{3}} d x_{2}^{2}+c^{3} d x_{3}^{2}(m \neq 0,-1),
$$

where again $a, b, c$ are functions on $x_{0}$ only. Like in the previous case, this model belongs to the spaces of diagonal curvature if $a=\lambda_{1} c, b=\lambda_{2} c$.

These examples show that some important known solutions of Einstein equations are the spaces of diagonal curvature. Note, that it is not clear so far how unique is diagonal coordinatization of Einstein spaces corresponding to the enlisted Bianchi models. It might happen that in some other diagonal coordinate system equations (3.10) are satisfied for a more broad class of spaces.

\section{Dressing method in application to spaces of diagonal curvature}

In this chapter we describe a procedure of integration of equations (3.10), a dressing method described in 1974 [2]. We present here some important developments of this method. 
Let $F\left(s, s^{\prime}, u\right)$ be a matrix $n \times n$-valued kernel of an integral operator $\hat{F}$ acting on vector-functions on the real axis $-\infty<s<\infty$, and $u=u^{1}, \ldots, u^{n}$ be $n$ dimensional parameter. One can study two simultaneous factorizations of the operator $\hat{F}$ :

$$
\begin{aligned}
& 1+\hat{F}=\left(1+\hat{K}^{+}\right)^{-1}\left(1+\hat{K}^{-}\right), \\
& 1+\hat{F}=\left(1+\hat{M}^{+}\right)\left(1+\hat{M}^{-}\right)^{-1}
\end{aligned}
$$

Here $\hat{K}^{ \pm}, \hat{M}^{ \pm}$are triangle operators. Their kernels, $K^{ \pm}\left(s, s^{\prime}, u\right)$ and $M^{ \pm}\left(s, s^{\prime}, u\right)$, satisfy the conditions:

$$
\begin{aligned}
& K^{+}\left(s, s^{\prime}\right)=0, \quad s^{\prime}<s, \\
& M^{+}\left(s, s^{\prime}\right)=0, s^{\prime}<s, \\
& K^{-}\left(s, s^{\prime}\right)=0, s^{\prime}>s, \\
& M^{-}\left(s, s^{\prime}\right)=0, s^{\prime}>s .
\end{aligned}
$$

Apparently,

$$
1+M^{+}=\left(1+K^{+}\right)^{-1},
$$

and $K^{+}, M^{+}$are connected by the relation:

$$
K^{+}\left(s, s^{\prime}\right)+M^{+}\left(s, s^{\prime}\right)+\int_{s}^{s^{\prime}} K^{+}\left(s, s^{\prime \prime}\right) M^{+}\left(s^{\prime \prime}, s^{\prime}\right) d s^{\prime \prime}=0 .
$$

In a half plane $s>s^{\prime}$, following integral equations hold:

$$
\begin{aligned}
& K^{+}\left(s, s^{\prime}\right)+F\left(s, s^{\prime}\right)+\int_{s}^{\infty} K^{+}\left(s, s^{\prime \prime}\right) F\left(s^{\prime \prime}, s^{\prime}\right) d s^{\prime \prime}=0, \\
& M^{-}\left(s, s^{\prime}\right)+F\left(s, s^{\prime}\right)+\int_{s^{\prime}}^{\infty} F\left(s, s^{\prime \prime}\right) M^{-}\left(s^{\prime \prime}, s^{\prime}\right) d s^{\prime \prime}=0 .
\end{aligned}
$$

Let $I_{i}$ be a set of projective operators acting in $n$-dimensional linear space and satisfying the conditions

$$
I_{i} I_{j}=I_{i} \delta_{i j}
$$

This set can be interpreted as a matrix,

$$
I_{i}=\operatorname{diag}(\underbrace{0, \ldots, 1}_{i} 0, \ldots, 0) .
$$

One can introduce a set of differential operators,

$$
D_{i} F=\frac{\partial}{\partial u^{i}}+I_{i} \frac{\partial F}{\partial s}+\frac{\partial F}{\partial s^{\prime}} I_{i},
$$


and require

$$
D_{i} F=0
$$

We assume also that $1+F$ is an invertible operator. In other words, the relation

$$
(1+F) \Omega=0
$$

implies $\Omega=0$. If conditions (5.9), (5.10) are fulfilled, the kernels $K^{+}$and $M^{-}$ satisfy equations

$$
\begin{aligned}
& \tilde{D}_{i} K^{+}=D_{i} K^{+}+\left[I_{i}, Q\right] K^{+}=0, \\
& \hat{D}_{i} M^{-}=D_{i} M^{-}+M^{-}\left[I_{i}, Q\right]=0,
\end{aligned}
$$

or

$$
\begin{aligned}
& \frac{\partial K^{+}}{\partial u^{i}}+I_{i} \frac{\partial K^{+}}{\partial s}+\frac{\partial K^{+}}{\partial s^{\prime}} I_{i}+\left[I_{i}, Q(s)\right] K^{+}\left(s, s^{\prime}\right)=0, \\
& \frac{\partial M^{-}}{\partial u^{i}}+I_{i} \frac{\partial M^{-}}{\partial s}+\frac{\partial M^{-}}{\partial s^{\prime}} I_{i}-M^{-}\left(s, s^{\prime}\right)\left[I_{i}, Q\left(s^{\prime}\right)\right]=0 .
\end{aligned}
$$

Here $Q(s)=K^{+}(s, s)=M^{-}(s, s)$. Moreover, matrix elements $Q_{i j}(s, u),(i \neq j)$ satisfy equation $(3.10)$,

$$
\frac{\partial Q_{i j}}{\partial u^{k}}=Q_{i k} Q_{k j}
$$

We will not prove this fact, which is almost trivial. Another two points are important:

1. Let $\phi_{i}=\phi_{i}\left(s-u_{i}\right)$ be a set of $n$ arbitrary functions of one variable. Then a set of functions,

$$
H_{i}(s, u)=\phi_{i}+\int_{s}^{\infty} K_{i}^{+}\left(s, s^{\prime}, u_{i}\right) \phi_{k}\left(s^{\prime}-u_{k}\right),
$$

presents the set of Lamé coefficients $H_{i}$ at any $s$. In other words, the metric

$$
d s^{2}=\sum_{i=1}^{n} H_{i}^{2}\left(d u^{i}\right)^{2},
$$

is a metric of certain space of diagonal curvature. A different choice of $\phi_{i}$ gives different Combescure equivalent metrics.

2. Let the following set of functions,

$$
\Psi_{i}\left(s^{\prime}, u\right)=\psi_{i}+\int_{s}^{\infty} \phi_{k}\left(s^{\prime}-u_{k}\right) M_{k_{1}}^{-}\left(s^{\prime}, s\right) d s^{\prime},
$$


be a set of adjoint Lamé coefficients satisfying equation (2.18). Then the metric

$$
d s^{2}=\sum_{i=1}^{N} \Psi_{i}^{2}\left(d u^{i}\right)^{2}
$$

presents the adjoint space of diagonal curvature. In Egorov's case, $F\left(s, s^{\prime}\right)$ satisfy the additional constrain,

$$
F_{i k}\left(s, s^{\prime}\right)=F_{k i}\left(s^{\prime}, s\right) .
$$

In this case $H_{i}=\Psi_{i}$.

\section{Differential reduction}

Following the approach of the dressing method, we will call $F\left(s, s^{\prime}, u\right)$, satisfying equation (5.8), a "dressing function". Relation (5.16) is an example of additional constrain, which could be imposed on $F$ without violation of basic equation (3.10). One can easily check that relation (5.16) implies the relation

$$
M^{-}\left(s, s^{\prime}\right)=\left[K^{+}\left(s^{\prime}, s\right)\right]^{t r},
$$

or

$$
M_{i j}^{-}\left(s, s^{\prime}\right)=K_{j i}^{+}\left(s^{\prime}, s\right)
$$

In this case,

$$
Q_{i j}(s, u)=Q_{j i}(s, u) .
$$

The additional relations imposed on $F$ can be called reductions. The key role in the theory of $n$-orthogonal coordinate systems plays the differential reduction,

$$
\frac{\partial F\left(s, s^{\prime}\right)}{\partial s^{\prime}}+\frac{\partial F^{t r}\left(s^{\prime}, s\right)}{\partial s}=0
$$

which was introduces in [8]. One can note that this reduction leads to an algebraic relation, which connects the kernels $K^{+}$and $M^{-}$. Also, it proves that differential constrain (6.4) stipulates the differential relation between $K^{+}$and $M^{-}$. Omitting a very simple proof, we will just formulate this relation:

$$
\left(\frac{\partial K^{+}\left(s^{\prime}, s\right)}{\partial s}\right)^{t r}+\frac{\partial M^{-}\left(s, s^{\prime}\right)}{\partial s^{\prime}}+M^{-}\left(s, s^{\prime}\right)\left[Q\left(s^{\prime}\right)-Q^{t r}\left(s^{\prime}\right)\right]=0 .
$$

Using this relation one can prove that on the diagonal $s=s^{\prime}$,

$$
\frac{\partial K^{+}\left(s, s^{\prime}\right)}{\partial s^{\prime}}+\left.\frac{\partial K^{+t r}\left(s^{\prime}, s\right)}{\partial s}\right|_{s^{\prime}=s}=-Q(s) Q^{t r}\left(s^{\prime}\right),
$$


and finally, that $Q_{i j}$ satisfy additional equations,

$$
\frac{\partial Q_{i j}}{\partial u^{j}}+\frac{\partial Q_{j i}}{\partial u^{i}}+\sum_{k \neq i, j} Q_{i k} Q_{j k}=0 .
$$

So, relation (6.4) is the reduction, which gives a solution for the problem of construction of $n$-orthogonal coordinate systems.

\section{One--soliton solution; a general case.}

The most striking point of the dressing method is an opportunity to construct exact solutions of integrable systems in a close form. Let us consider a general case of spaces of diagonal curvature and suppose that the "dressing" function $F$ is a product of two matrixes:

$$
F\left(s, s^{\prime}, u\right)=A(s-u) B\left(s^{\prime}-u\right) .
$$

We will call the corresponding solution of equation (3.19) as "one-soliton solution". In fact, this is a very complicated solution, which includes many interesting Riemann spaces. To satisfy the basic condition (5.8) one should put

$$
\begin{aligned}
& A_{i j}=A_{i j}\left(s-u^{i}\right), \\
& B_{i j}=B_{i j}\left(s^{\prime}-u^{j}\right) .
\end{aligned}
$$

Integral equations (5.5) and (5.6) can be solved immediately:

$$
\begin{aligned}
& K^{+}\left(s, s^{\prime}\right)=K^{+}(s) B\left(s^{\prime}\right)= \\
& M^{-}\left(s, s s^{\prime}\right)=A(s) M^{-}\left(s^{\prime}\right) .
\end{aligned}
$$

Here

$$
\begin{aligned}
& K^{+}(s)=-A(s)(1+\Delta(s))^{-1}, \\
& M^{-}\left(s^{\prime}\right)=-\left(1+\Delta\left(s^{\prime}\right)\right)^{-1} B\left(s^{\prime}\right), \\
& \Delta_{i k}=\sum_{p} \int_{-\infty}^{u^{p}-s} B_{i p}(\xi) A_{p k}(\xi) d \xi .
\end{aligned}
$$

The rotation coefficients $Q_{i j}$ take the form

$$
\begin{aligned}
Q_{i j} & =Q_{i j}\left(u^{1}-s, \cdots, u^{n}-s\right)= \\
& =-\left.A_{i p}\left[(1+\Delta)^{-1}\right]_{p q} B_{q j}\right|_{s=s^{\prime}}
\end{aligned}
$$


In this equation one can put $s=0$. In a general case, $Q_{i j}$ is parametrized by $2 n^{2}$ functions of one variable.

Let $f_{i}=f_{i}\left(u^{i}-s\right)$ be a set of arbitrary functions of one variable. All Combescure equivalent metrics of spaces of diagonal curvature corresponding to the rotation coefficients (7.6) are given as

$$
H_{i}=f_{i}-A_{i p}\left[(1+\Delta)^{-1}\right]_{p q} R_{q}
$$

where

$$
R_{q}=\sum_{k} \int_{-\infty}^{u^{k}-s} B_{q k}(\xi) f_{k}(\xi) d \xi .
$$

For the given $Q_{i j}$, they are parametrized by functions $f_{n}$.

The adjoint Lamé coefficients $\Psi_{i}$ are parametrized by another set of functions on one variable, $g_{k}\left(u^{k}-s\right)$. Now

$$
\begin{aligned}
\Psi_{i} & =g_{i}-T_{p}\left[(1+\Delta)^{-1}\right]_{p q} B_{q i} \\
T_{p} & =\sum_{k} \int_{-\infty}^{u^{p}-s} g_{k}(\xi) A_{k p}(\xi) d \xi .
\end{aligned}
$$

A general solution of the Laplace equations (2.21), (2.22) is given in the form

$$
h=h_{0}-T_{p}\left[(1+\Delta)^{-1}\right] R_{q},
$$

where

$$
h_{0}=\sum_{k} \int_{-\infty}^{u^{k}-s} f_{k}(\xi) g_{k}(\xi) d \xi .
$$

Diagonal elements of the curvature tensor can be presented in the form

$$
\begin{aligned}
E_{i j}= & \frac{\partial Q_{i j}}{\partial u^{j}}+\frac{\partial Q_{j i}}{\partial u^{i}}+\sum_{k \neq i, j} Q_{i k} Q_{j k}= \\
= & -A_{i p}\left[(1+\Delta)^{-1}\right]_{p q} B_{q j}^{\prime}-A_{j p}\left[(1+\Delta)^{-1}\right]_{j p} B_{q i}^{\prime}+ \\
& +A_{i p} A_{j k}\left[(1+\Delta)^{-1}\right]_{p l}\left[(1+\Delta)^{-1}\right]_{q m} B_{l k} B_{m k}
\end{aligned}
$$

\section{One--soliton solutions; special cases.}

One-soliton solutions exist for all special classes of spaces of diagonal curvature. They are separated from general one-soliton solutions by imposing of some constrain on matrixes $A(s), B(s)$. 
The most interesting case is a flat Euclidean space $R^{n}$. In this case $A$ and $B$ are connected:

$$
A=B^{t r^{\prime}} \Lambda \text { or } A_{i j}=B_{k i}^{\prime} \Lambda_{k j} .
$$

Here $\Lambda_{k j}=-\Lambda_{j k}$ are constant antisymmetric matrixes. One can check directly (while it is a hard procedure) that, if conditions (8.1) are satisfied, $E_{i j}=0$ and the metric is flat.

The problem of embedding of the $n$-orthogonal metric to $R^{n}$ can be solved efficiently in the soliton case. Suppose, that the potential $h$ satisfying the Laplace equation (2.23), satisfy also the equation

$$
\frac{\partial^{2} h}{\left(\partial u^{l}\right)^{2}}=\sum_{k} \Gamma_{l l}^{k} \frac{\partial h}{\partial u^{k}} .
$$

In virtue of (2.20), equation (8.2) is equivalent to the relation

$$
\frac{\partial \Psi_{l}}{\partial x_{l}}=-\sum_{k \neq l} Q_{l k} \Psi_{k},
$$

which is compatible with the definition of $\Psi_{l}$

$$
\frac{\partial \Psi_{l}}{\partial u^{k}}=Q_{l k} \Psi_{k}
$$

if and only if the metric is flat and $E_{i k} \equiv 0$.

Apparently, equation (8.4) is satisfied only for a very special choice of function $g_{i}(\xi)$. The following theorem holds:

Theorem 1. Equations (8.3), (8.4) are satisfied if and only if $g_{i}(\xi)$ are constants.

The proof of this theorem is straightforward but cumbersome. It will be published in another article. It should be noted that solutions $h$ in (2.23), (8.3) are defined up to an arbitrary constant. If one takes $n-1$ arbitrary common solution of this system, equations

$$
h^{i}\left(u^{1}, \cdots, u^{n}\right)=c^{i}, \quad i=1, \cdots, n-1,
$$

define all possible geodesics (straight lines) in $R^{n}$. By a proper orthogonalization we could define $x^{i}=x^{i}\left(u^{1}, \cdots, u^{n}\right)$ and accomplish introducing of $n$-orthogonal coordinates for the soliton case. In the same way, one can find the constrains connecting $A$ and $B$ for the spaces of constant curvature and for the spaces of flat connection. 
It is a much more difficult problem to determine reductions, which separate solutions of Einstein equations for the given equation of matter state. We discuss here the vacuum case only. In this case one should satisfy equations (4.10), (4.15).

Let us study an infinitesimal dressing $F \rightarrow 0$. In this limit

$$
Q\left(u^{1}-s, \cdots, u^{n}-s\right)=K(s, s)=-F(s, s),
$$

and

$$
-E_{i j}=\frac{\partial F_{i j}\left(u^{i}-s, u^{j}-s^{\prime}\right)}{\partial s^{\prime}}+\frac{\partial F_{j i}\left(u^{j}-s^{\prime}, u^{i}-s\right)}{\partial s} .
$$

At $s=0, E_{i j}=E_{i j}\left(u^{i}, u^{j}\right)$. According to (4.10) and (4.15), $E_{i j}=\alpha_{i j} H_{i} H_{j}$, and

$$
\alpha_{12}=\alpha_{34}=\alpha, \alpha_{13}=\alpha_{23}=\beta, \quad \alpha_{14}=\alpha_{23}=\gamma \text {. }
$$

As far as we have $H_{i}=H_{i}\left(u^{i}\right), H_{j}=H_{j}\left(u^{j}\right)$ in the linear approximation, $\alpha_{i j}$ must be constants. Finally, the dressing matrix $F$ should satisfy the condition

$$
\frac{\partial F_{i j}\left(u^{i}-s, u^{j}-s^{\prime}\right)}{\partial s^{\prime}}+\frac{\partial F_{j i}\left(u^{j}-s^{\prime}, u^{i}-s\right)}{\partial s}=\alpha_{i j} H_{i}\left(u^{i}-s\right) H_{j}\left(u^{j}-s^{\prime}\right),
$$

where $\alpha_{i j}=\alpha_{j i}$ is a symmetric constant matrix satisfying conditions (4.15).

One must remember that conditions (8.8) are necessary. The sufficient conditions, which must be imposed on $F$ to find the exact solution of Einstein equations in vacuum, are unknown so far.

\section{Dressing via $\bar{\partial}-$ problem}

The dressing procedure described above will lead to the same results, if one performs the following replacement in (5.5), (5.6):

$$
\int_{s}^{\infty} u d s \rightarrow \frac{1}{2}\left(\int_{s}^{\infty} u d s-\int_{-\infty}^{s} u d s\right)
$$

Let us indroduce:

$$
\begin{aligned}
F\left(s, s^{\prime}\right) & =\int F(\lambda, \mu) e^{-i\left(\lambda s-\mu s^{\prime}\right)} d \lambda d \mu, \\
K^{+}\left(s, s^{\prime}\right) & =\int K(s, \mu) e^{i \mu s^{\prime}} d \mu, \\
M^{+}\left(s, s^{\prime}\right) & =\int M\left(\lambda, s^{\prime}\right) e^{-i \lambda s} d \lambda .
\end{aligned}
$$


One can check that equations (5.5), (5.6) are equivalent to integral equations:

$$
\begin{aligned}
\phi(s, \lambda)+\int_{-\infty}^{\infty} f(\eta, \lambda) d \eta-i \int_{-\infty}^{\infty} \frac{\phi(s, \xi)}{\eta-\xi} f(\eta, \lambda) d \xi d \eta & =0, \\
\psi\left(\lambda, s^{\prime}\right)+\int_{-\infty}^{\infty} f(\lambda, \eta) d \eta+i \int_{-\infty}^{\infty} \frac{f(\lambda, \xi)}{\xi-\eta} \psi\left(\eta, s^{\prime}\right) d \xi d \eta & =0,
\end{aligned}
$$

where

$$
\begin{aligned}
\phi(s, \lambda) & =K(s, \lambda) e^{i \lambda s}, \\
\psi\left(\lambda, s^{\prime}\right) & =M\left(\lambda, s^{\prime}\right) e^{-i \lambda s^{\prime}}, \\
f(\lambda, \eta) & =F(\lambda, \eta) e^{i(\lambda-\eta) s} .
\end{aligned}
$$

Later on we will omit sometimes the notations $s, s^{\prime}$. In equations (9.5), (9.6)

$$
\frac{1}{\eta-\xi}=\lim _{\epsilon \rightarrow 0} \frac{\bar{\eta}-\bar{\xi}}{|\eta-\xi|^{2}+\epsilon^{2}}
$$

Taking into account (9.8), one can understand the integration performed in (9.5), (9.6) in a more broad sense. So far, all functions are defined in the real axis $-\infty<\lambda<\infty,-\infty<\eta<\infty$. The dressing procedure does not change if one assumes that $\phi, \lambda$ are quasianalytic functions defined on the whole complex plane $C^{1}$. In this case, $F(\eta, \lambda)$ is defined on $C^{2}$. One can define:

$$
\begin{aligned}
\chi(\lambda, \bar{\lambda}) & =1-i \int \frac{\phi(\xi, \bar{\xi})}{\lambda-\xi} d \xi d \bar{\xi} \\
\bar{\xi}(\lambda, \bar{\lambda}) & =1+i \int \frac{\psi(\eta, \bar{\eta})}{\lambda-\eta} d \eta d \bar{\eta} .
\end{aligned}
$$

Using the standard formula,

$$
\frac{\partial}{\partial \bar{\lambda}} \frac{1}{\lambda}=\pi \delta(\lambda) \delta(\bar{\lambda})
$$

one obtains

$$
\phi(\lambda, \lambda)=-\frac{1}{\pi i} \frac{\partial \chi}{\partial \bar{\lambda}}, \quad \psi(\lambda, \bar{\lambda})=\frac{1}{\pi i} \frac{\partial \tilde{\chi}}{\partial \lambda} .
$$

Quasianalytic functions $\chi, \tilde{\chi}$ satisfy the equations:

$$
\begin{aligned}
& \frac{\partial \chi}{\partial \bar{\lambda}}=-\pi i \int \chi(\xi, \bar{\xi}) f(\xi, \bar{\xi}, \lambda \bar{\lambda}) d \xi d \bar{\xi} \\
& \frac{\partial \bar{\chi}}{\partial \lambda}=\pi i \int f(\lambda, \bar{\lambda}, \xi, \bar{\xi}) \bar{\chi}(\xi, \bar{\xi}) d \xi d \bar{\xi}
\end{aligned}
$$


which define the dual "non-local $\bar{\partial}$-problem", and are accomplished by the following normalization:

$$
\chi \rightarrow 1, \quad \bar{\chi} \rightarrow 1 \quad \text { at } \quad \lambda \rightarrow \infty .
$$

In the limit $\lambda \rightarrow \infty$ one has:

$$
\begin{aligned}
\chi & =1+\frac{Q}{i \lambda}+\frac{P}{(i \lambda)^{2}}+\frac{R}{(i \lambda)^{2}}+\cdots \\
Q & =K^{+}(s, s)=\int K(s, \mu) e^{i \mu s} d \mu=\int \phi(\xi, \bar{\xi}) d \xi d \bar{\xi} \\
P & =\left.\frac{\partial K}{\partial s}\right|_{s=s^{\prime}}=i \int \xi \phi(\xi, \bar{\xi}) d \xi d \bar{\xi}
\end{aligned}
$$

In the same way,

$$
\tilde{\chi}=1-\frac{Q}{i \lambda}+\frac{\tilde{P}}{(i \lambda)^{2}}+\cdots .
$$

Equation (5.8) imposes on function $f(\lambda, \eta)$ the following condition:

$$
\begin{aligned}
f(\lambda, \eta) & =e^{i \lambda \Phi} F(\lambda, \eta) e^{-i \eta \Phi} \\
\Phi & =s+\sum_{i=1}^{n} I_{i} u^{i} .
\end{aligned}
$$

In virtue of (9.12) and (9.13), functions $\chi, \tilde{\chi}$ satisfy the linear systems:

$$
\begin{aligned}
& I_{k}\left(\frac{\partial \chi}{\partial u^{i}}+i \lambda \chi I_{i}-Q I_{i} \chi\right)=0, \quad i \neq k, \\
& \left(\frac{\partial \tilde{\chi}}{\partial u^{i}}-i \lambda I_{i} \tilde{\chi}-\tilde{\chi} I_{i} Q\right) I_{k}=0, \quad i \neq k .
\end{aligned}
$$

Let us expand equation (9.20) in powers of $1 / i \lambda$. The first term of the expansion reads:

$$
I_{k} \frac{\partial Q}{\partial u^{i}}-I_{k} Q I_{i} Q+I_{k} P=0 .
$$

Multiplication by $I_{j}(j \neq i, k)$ from the right side gives

$$
I_{k} \frac{\partial Q}{\partial u^{i}} I_{j}=I_{k} Q I_{i} Q I_{j}
$$

This is just another notation for equation (5.13). Multiplication of (9.22) by $I_{i}$ leads to definition of $P$,

$$
I_{k} P I_{i}=I_{k} Q I_{i} Q I_{i}-I_{k} \frac{\partial Q}{\partial u^{i}} I_{i}
$$


The second term of the expansion leads to the equation:

$$
I_{k} \frac{\partial P}{\partial u^{i}} I_{i}=I_{k} Q I_{i} P I_{j}
$$

Let us introduce

$$
\chi=X e^{-i \lambda \Phi}, \quad \tilde{\chi}=e^{i \lambda \Phi} Y .
$$

Apparently, $X$ and $Y$ satisfy equations

$$
\begin{aligned}
I_{k} \frac{\partial X}{\partial u^{i}} & =I_{k} Q I_{i} X, \\
\frac{\partial Y}{\partial u^{k}} I_{i} & =Y I_{i} Q I_{k} .
\end{aligned}
$$

Suppose, that $\phi_{i}=\phi_{i}(\lambda, \bar{\lambda}), \quad \psi_{i}=\psi_{i}(\lambda, \bar{\lambda})$ are two arbitrary sets of functions of some complex variable, not necessary analytical one. One can see that the sets

$$
\begin{aligned}
H_{i}\left(u^{1}, \ldots, u^{n}\right) & =\sum_{k} \int X_{i k}\left(u^{1}, \ldots, u^{n}, \xi, \bar{\xi}\right) \phi_{k}(\xi, \bar{\xi}) d \xi d \bar{\xi} \\
\Psi_{i}\left(u^{1}, \ldots, u^{n}\right) & =\sum \int Y\left(u^{1 *}, \ldots, u^{n}, \xi, \bar{\xi}\right) \psi_{i}(\xi, \bar{\xi}) d \xi d \bar{\xi}
\end{aligned}
$$

give the arrays of Lamé coefficients and adjoint Lamé coefficients.

Now the reductions are some restrictions imposed on $f(\lambda, \eta)$. The fundamental reduction (6.4) reads:

$$
\eta F(\lambda, \eta)=\lambda F^{t r}(-\eta,-\lambda) .
$$

Let us impose a more general reduction,

$$
\eta F(\lambda, \eta)-\lambda F^{t r}(-\eta,-\lambda)=e^{i \lambda \Phi} R(\lambda, \eta) e^{-1 \lambda \Phi},
$$

where

$$
R(\lambda, \eta)=R^{\operatorname{tr}}(-\eta,-\lambda)
$$

is some matrix function on $C^{2}$ and does not depend on $u^{i}$. From (9.12) one gets:

$$
\frac{\partial}{\partial \bar{\lambda}} \chi^{t r}(-\lambda,-\bar{\lambda})=\pi i \int f^{t r}(-\xi,-\bar{\xi},-\lambda,-\bar{\lambda}) \chi^{t r}(-\xi,-\bar{\xi}) d \xi d \bar{\xi}
$$

Using (9.12), (9.32) and (9.30) one can obtain the following bilinear identity:

$$
\begin{aligned}
& \int \frac{\partial}{\partial \bar{\lambda}} \lambda \chi(\lambda, \bar{\lambda}) \chi^{t r}(-\lambda,-\bar{\lambda}) d \lambda d \bar{\lambda}= \\
& -\pi i \int \chi(\xi, \bar{\xi}) e^{i \xi \Phi} R(\xi, \eta) e^{-i \eta \Phi} \chi^{t r}(-\eta,-\bar{\eta}) d \xi d \bar{\xi} d \eta d \bar{\eta}
\end{aligned}
$$


The integral in the left part of (9.34) is proportional to the residue of $\lambda \chi \chi^{t r}$ at infinity. Using the asymptotic expansion (9.14) one gets:

$$
\int \frac{\partial}{\partial \bar{\lambda}} \lambda \chi(\lambda, \bar{\lambda}) \chi^{t r}(-\lambda,-\bar{\lambda}) d \lambda d \bar{\lambda}=2 \pi i\left(P-P^{t r}-Q Q^{t r}\right) .
$$

Suppose, that $R(\lambda, \eta)$ is presented in the form:

$$
R_{i j}(\lambda, \eta)=-2 \sum_{k=1}^{N} \phi_{i}^{(k)}(\lambda, \bar{\lambda}) \phi_{j}^{(k)}(-\eta,-\bar{\eta})(-1)^{\alpha(k)} .
$$

Here $\phi_{i}^{(k)}(\lambda, \bar{\lambda}), i=1, \ldots, N$ are some functions on $C^{1}$, and $\alpha(k)=0,1$. Then formula (9.34) reads:

$$
P-P^{t r}-Q Q^{t r}=\sum_{k \pm 1}^{N} H_{i}^{(k)} H_{j}^{(k)}(-1)^{\alpha(k)} .
$$

Here

$$
H_{i}^{k}=\sum_{q} \chi_{i q}(\xi, \bar{\xi}) e^{i \xi u^{k}} \Psi_{q}^{k}(\xi, \bar{\xi}) d \xi d \bar{\xi}
$$

$H_{i}^{k}$ are the arrays of Combescure equivalent metrics at different $k$. Combining (9.36) with (9.35), one easily obtains:

$$
E_{i j}=\sum_{k} H_{i}^{(k)} H_{j}^{(k)}(-1)^{\alpha(k)} .
$$

In other words, reduction (9.31) describes a space of flat connection. If $N \rightarrow \infty$, one can present any matrix function $R_{i j}(\lambda, \eta)$ in the form (9.36). That means that the spaces of flat connection are dense in the set of all spaces of diagonal curvature.

There are some other, more sophisticated methods that allow to separate the spaces of flat connection from the total pool of diagonal curvature spaces. One of them is describes in [14].

Most probably, the Einstein spaces of diagonal curvature are among the spaces of flat connection.

\section{References}

1. D. Kaup, The three-wave interaction - a non-dispersive phenomenon. Studies Appl. Math., 55 (1976), pp. 9-44. 
2. V. Zakharov and A. Shabat, An integration scheme for the nonlinear equations of mathematical physics by the method of the Inverse scattering problem. Funk. Anal. Appl., 8 (1974), pp. 43-54, 226-235.

3. V. Zakharov and S. Manakov, The theory of resonant interaction of wave packets in nonlinear media. Zh. Eksper. Teor. Fiz., 69 (1975), pp. 16541673; Soviet Phys. JETP, 42 (1976), pp. 842-850.

4. G. Darboux, Lecons sur le Systemes Orthogonaux et les Coordonnees Curvilignes, Gauthier-Villars, Paris, 1910.

5. B. Dubrovin, Integrable systems in topological field theory. Nuclear Phys., B 379 (1992), pp. 627-689; On the differential geometry of strongly integrable systems of hydrodynamic type, Funk. Anal. Appl. 24 (1990), pp. 280-285.

6. B. Dubrovin and S. Novikov, Hamiltonian formalism of one-dimensional systems of hydrodynamic type and the Bogolyubov-Whitham averaging method. Dokl. Acad. Nauk SSSR 270 (1983), pp. 781-785.

7. S. Tsarev, Poisson's brackets and one-dimensional Hamiltonian systems of hydrodynamic type, Dokl. Akad. Nauk SSSR, 282 (1985), pp. 534-537.

8. V. Zakharov, Description of $n$-orthogonal curvilinear coordinate systems and Hamiltonian integrable systems of hydrodynamic type. Duke Mathematical J., 94:1 (1998), pp. 103-139.

9. V. Zakharov and S. Manakov, Reductions in systems integrable by the method of inverse scattering problem. Doclady Math. 57:3 (1998), pp. 471-474.

10. V. Zakharov, How classical physics helps mathematics. GAFA Geometrical and Functional Analysis, Special Volume GAFA 2000, pp. 859-879.

11. E. Ferapontov, Private communication.

12. V. Zakharov, On the dressing method, in "Inverse methods in action", Springer-Verlag, Berlin, 1990, pp. 602-623.

13. V. Zakharov and S. Manakov, Construction of multidimensional nonlinear integrable systems and their solitons. Funk. Anal. Appl. 19 (1985), pp. $11-25$.

14. V. Zakharov, Integrating of the Gauss-Codazzi equations. Theor. Math. Phys. 128 (2001), pp. 946-956. 